\title{
ETHICAL ASPECTS OF PREVENTION OF OVERWEIGHT AND OBESITY
}

\section{R. Goranova-Spasova*, N. Shtereva-Nikolova, A. Traykovska-Dimitrova}

\author{
Faculty of Public Health, Medical University- Sofia, Sofia, Bulgaria
}

\begin{abstract}
Obesity is one of the leading preventable causes of death in the world. The prevalence of obesity among adults and children is one of the most serious public health issues in the 21st century. According to the World Health Organization (WHO) in 2016, more than 1.9 billion adults are overweight. Over 650 million of them are obese. 41 million children under the age of 5 were overweight or obese.

PURPOSE: The aim of our study is to examine and analyze the ethical acceptability of obesity prevention approaches worldwide.

METHODS: In order to achieve this goal, we have used general and private science methods and approaches, including documentary method, analysis and synthesis, comparative method.

RESULTS: Prevention of obesity can be done at individual and population level with the emphasis on public health initiatives. The analysis of obesity prevention approaches shows that there is a risk that the program may lead to stigmatization of a particular group, victim blaming and reinforcement of health inequalities.

CONCLUSION: The implementation of a public health initiative should be based on evidence of its effectiveness but also on ethical considerations to ensure the protection of human rights
\end{abstract}

Key words: ethical approach, public health intervention, health programs, obese

\section{INTRODUCTION}

Obesity is defined as a disease state where the body mass index is 30 or higher. The causes of obesity are multiple, most often a combination of excessive energy intake, lack of physical activity and genetic predisposition. Among the factors should also be mentioned endocrine diseases, mental illness and side effects of medication. (1)

The World Health Organization (WHO) classifies body mass index obesity (BMI) as follows:

- $\quad<18.5$ - Underweight

- 18.5-24.9- Normal weight

- 25.0-29.9 - Overweight

- 30.0-34.9- Class I obesity

- 35.0-39.9- Class II obesity

- $\quad \geq 40$ - Class III obesity

Obesity is one of the leading preventable causes of death in the world. (1) The spread of obesity among adults and children puts it

*Correspondence to: Radka Nikolaeva GoranovaSpasova, 8 Byalo more str., 1527 Sofia, Bulgaria +359898240976, e-mail: r.goranova@foz.musofia.bg among the most serious public health problems in the 21st century. According to data from WHO in 2016, more than 1.9 billion adults worldwide are overweight. Of these, over 650 million have obesity. For the same year, 41 million children under the age of 5 were overweight or obese, and over 340 million children and adolescents aged 5 to 19 were overweight. (2) So these presented data suggests that obesity is becoming a pandemic. In the United States, one of the most affected country, statistics show that one in three adults and one in six children have some degree of overweight or obesity. (3) The problem affects not only high-income countries, but also lowand middle-income countries, especially in the urban environment.

According to data from the National Center for Public Health and Analysis (NCPHA) overweight and obesity are a serious problem in our country, with $5 \%$ of the children under the age of 4 being overweight and among students it reaches $30 \%$. From $12 \%$ to $15 \%$ of children are obese. (4) 
The health consequences of overweight and obesity are multiple. Obesity increases the risk of some chronic noninfectious diseases: cardiovascular diseases (mainly ischemic heart disease and stroke), type 2 diabetes mellitus, osteoarthritis, some cancers (endometrial, ovarian, breast, prostate, gallbladder, kidney and the colon) (5-6). In addition to the direct physical health consequences, the psychological, emotional and social effects of overweight can not be overlooked.

\section{PURPOSE OF THE STUDY}

The purpose of this paper is to examine and analyze the ethical acceptability of existing global approaches to the prevention of overweight and obesity.

\section{MATERIALS AND METHODS}

In order to achieve this goal, we used general and scientific methods and approaches,
GORANOVA-SPASOVA R., et al. including documentary method, analysis and synthesis, comparative method, induction and deduction. These methods complement each other.

\section{RESULTS AND DISCUSSION ACTIVITIES TO CONTROL THE OBESITY}

Figure 1 presents an Ishikawa Diagram illustrating the factors for obesity. The Ishikawa chart (or "fishbone" chart) is a causal diagram that visualizes the causes of a problem, as well as the interaction of these causes. In this case, the problem is "obesity" to the right, and the main reasons for its occurrence are on the horizontal axis. The main categories in it most often correspond to the factors influencing the process - people, technology, material, methods.

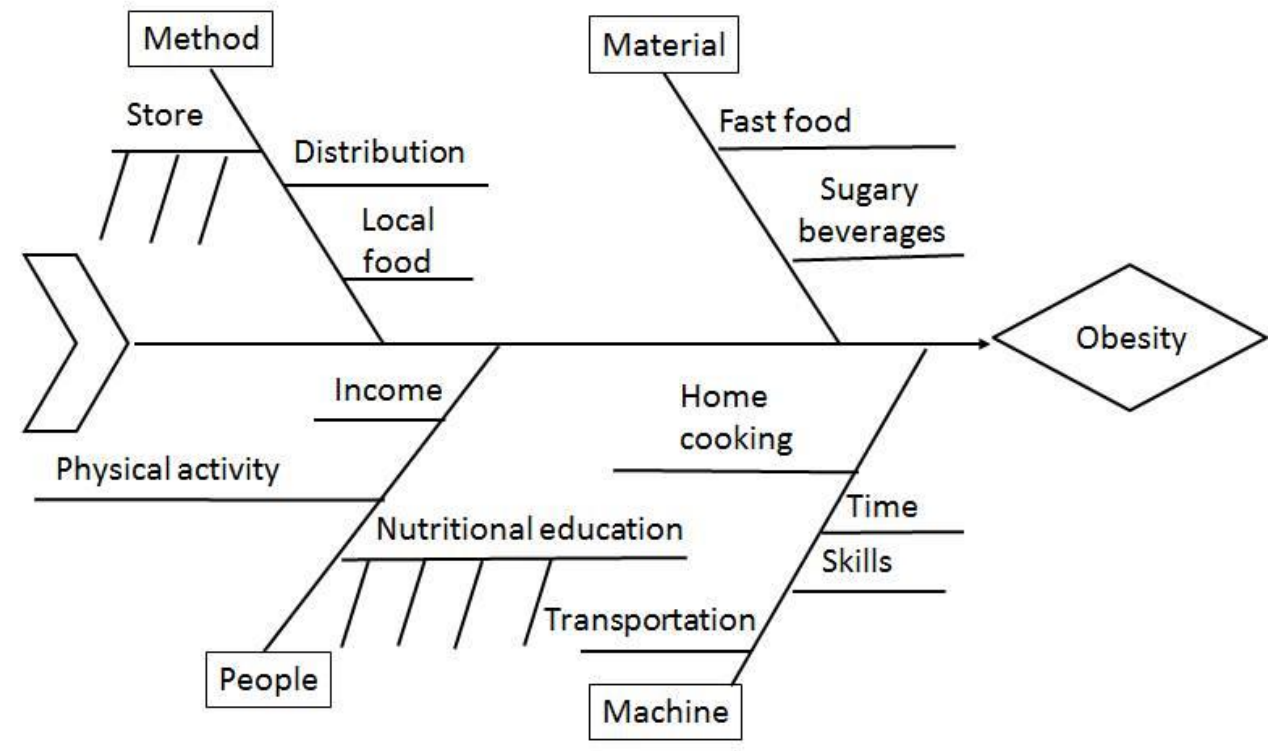

Figure 1. Ishikawa Diagram of Obesity (adapted from OFD Institute (2012)

Overweight and obesity control activities are aimed to modify these factors. The goals are: limiting over-consumption of high-energy foods and drinks; elimination of factors for reduced physical activity; nutrition education (7); promoting a healthy lifestyle; market control over the quality, safety and price of food, etc. Individual factors, apart from physical activity, are also income and culture in relation to nutrition. The adolescents are a specific risk group because feeding in childhood and young age is predestinating for the model of nutrition and in adulthood. Target groups for action are those with lower socioeconomic status and children. (8) Efforts are aimed at enhancing the health culture, reducing the harmful food and drink in kindergartens and schools, and creating circumstances for sports. (9-11)

As mentioned earlier, the factors of obesity are multiple, and therefore the approaches for dealing with it must be complex. In the United Kingdom, the obesity program for the prevention, identification, and management of overweight and obesity in adults and children distinguishes "upstream" "central" and "downstream" obesity prevention policies. "Upward" policies are aimed at the whole of society, the "central" of the individual's behavior, and the "downward" to the treatment of the people already affected. (12)

Numerous studies have shown that interventions at individual level do not provide 
the expected results for limiting obesity morbidity, so emphasis is placed on public health (13-14).

\section{INITIATIVES TO FIGHT OBESITY IN EUROPE}

The European Union is actively involved in the fight against obesity. One of the main ways in which the Commission does so is through the Europe Strategy on nutrition, obesity and obesity related health issues (2007) and the instruments for its implementation - the High Level Group on Nutrition and Physical Activity and the EU Platform for Action on Nutrition, Physical Activity and Health. The aim of the White Paper is to propose an integrated EU approach to reducing health problems due to improper diet, overweight and obesity. (15). The strategy focuses on proposing actions that can be carried out at local, regional, national and EU level by different partners in order to tackle this public concern. These actions complement and support existing measures in EU countries. The emphasis is on the socio-economic dimension of the obesity problem because of its association with less populous groups of society.

Other strategic documents outlining the future nutrition policy are:

- WHO European Surveillance on Obesity in Children (COSI), 2016 (WHO, with EU support, initiated the creation of a European Child Abuse Observation Network) 23 countries are participating in the project to create a European database for the spread of overweight and obesity in children for food policy purposes);

- The European Action Plan on Obesity in Children, 2014-2020;

- The European Food and Food Action Plan, 2015-2020. (16)

\section{Priority activities include:}

- Reducing the pressure of marketing and advertising of food and drink for children;

- Providing easy-to-understand nutrition information in food labeling;

- Reformulation of processed foods to reduce the salt, sugar and fat content;

- Promotion of healthy eating for infants and young children;

- Encourage and protect exclusive breastfeeding and provide healthy food at school.

Most premature deaths from chronic noncommunicable diseases can be prevented by integrating health into all policies with broad stakeholder involvement, government and
GORANOVA-SPASOVA R., et al. society. The policy package aims to improve supply and access to healthy food for the population and includes: creating conditions for choosing healthy foods and drinks; promoting the benefits of healthy lifelong learning, especially for the most vulnerable groups; Strengthening health systems to promote healthy eating; support for monitoring, evaluation and research; strengthening governance to ensure a health approach across all policies. (4)

A new Food Law is forthcoming, where all the new European standards, including those that Bulgaria has added in its legislation, are very detailed. (16)

Food control in the country is carried out by the Ministry of Agriculture and Food, the Ministry of Health, the Food Safety Agency.

\section{HEALTHY EATING PROGRAMS}

Bulgaria has developed a National Program for the Prevention of Chronic Non-communicable Diseases 2014-2020, which includes the risk factors - unhealthy nutrition, smoking and alcohol, as well as a detailed regulatory framework. (17-18)

From 2017 two programs for healthy eating are applied to students - "School Fruit" and "School Milk". (Ordinance on the conditions and the procedure for implementation of schemes for supplying fruit and vegetables and milk and milk products in educational establishments - School Fruit Scheme and School Milk Scheme). The Ministry of Health regulates nutrition for children in different age groups separate regulations:

- Ordinance No 37 of 21.07. 2009 for healthy eating of students;

- Ordinance No. 9 of 16.09 .2011 on the specific requirements for food safety and quality offered in kindergartens, school chairs and retail establishments on the territory of schools and children's establishments as well as on foods offered at organized events for children and students (Title, SG No. 60/2012, amended, No. 85 of 2015, in force from 3.11.2015)

- Ordinance No. 6 of 10.08. 2011 for healthy eating of children aged 3 to 7 years in childcare facilities.

\section{PUBLIC-HEALTH APPROACHES TO THE PREVENTION OF OBESITY}

An important question to be answered as a priority when developing a program for the prevention of overweight and obesity is: Can people really make choices about their diet and physical activity? And if the answer is "no", 
then who is responsible for spreading this public health problem? (19)

The rigorous implementation of a promotion or prevention program should be based on evidence of its effectiveness but also on ethical considerations. The "non-harm" principle is leading in the development of initiatives in the field of public health. (20) This guarantees the protection of human rights. The balance between the common and the individual good must always be respected.

Azavedo \& Vartanian (2015) examine the ethical acceptability of public health approaches in three areas (21):

1. Changes in the social-communication environment (raising awareness through food labeling, nutrition of fast food, etc., shifting focus from obesity to healthy lifestyle);

2. Changes in the economic environment (fees for harmful food and drink);

3. Changes in the physical environment / access (access to quality, safe food, limited access to detrimental food for adolescents, creating conditions for sports).

The review of literature and the analysis of obesity prevention approaches indicate that there is a risk that the program will lead to:

- Stigmatization of individual individuals or groups when the individual is stigmatized and rejected by the group (22);

- Limiting the autonomy of the individual when free choice is limited at the expense of the paternalistic model (23-24);

- Accusing the "victim" when an individual suffers an additional burden of choice that he has not done;

- The opposite effect of the program - in the case of the individual who wants to be dissociated from the whole;

- Enhancing health inequalities disadvantaged groups because of the imposition of a tax on "harmful food" will not be able to afford what they had so far (25).

The authors' conclusions are that activities aimed at changing access to harmful food and drink stimulate all people, not just those with obesity, to lead a healthy lifestyle. As a result, they are most ethically acceptable.

Ethical criticism of legislative measures to control access to certain harmful foods by prohibiting or charging taxes and duties is that they violate personal choices, restrict personal freedoms in a very paternalistic way, and stigmatize certain groups of the population.

Vartanian \& Syith (2013) discuss the ethical acceptability of the "stigmatization of people with obesity" approach. This approach is based on two hypotheses: 1. Obesity is subject to self-control; 2. Stigma on obese individuals will motivate them to change their lifestyle. Empirical data, as the authors find, does not support these hypotheses. They do not take into account the wide range of bio-psychosocial factors for overweight. Initiatives based on this approach are not subject to the basic principle of medical ethics - not to be harmed first (20).

\section{AN ETHICAL FRAMEWORK FOR PREVENTING OVERWEIGHT AND OBESITY}

ten Have et all. (2013) develop an ethical framework for the prevention of obesity and obesity, basing it on the eight starting points that a program should observe to be ethically acceptable.

1. Effects on physical health;

2. Psychosocial well-being;

3. Informed choice;

4. Cultural values;

5. Equality;

6. Respect for privacy;

7. Responsibility;

8. Freedom.

The framework proposed by the authors is a tool for systematic ethical evaluation. (26)

\section{CONCLUSION}

Due to the high importance of obesity as a public health issue, initiatives to manage it are imperative globally. The widespread application of an initiative must be based on evidence of its effectiveness but also on ethical considerations to ensure the protection of human rights. The analysis of existing approaches gives priority to approaches aimed at modifying the physical environment and access to food. Approaches affecting the entire population, not just obese individuals, and focusing on healthy lifestyles, are potentially less ethical.

\section{REFERENCES}

1. World Health Organization (WHO). Obesity and overweight: Fact sheet N 3111. 2015.

$<$ http://www.who.int/mediacentre/factsheet s/fs311/en/>.

2. Obesity and overweight: key facts, accessed on 23.06.2018<http://www.who.int/newsroom/fact-sheets/detail/obesity-andoverweight> 
3. Kass N, Hecht K, Paul A, Birnbach K. Ethics and obesity prevention: ethical considerations in 3 approaches to reducing consumption of sugar-sweetened beverages. Am J Public Health. May;104(5):787-95, 2014.

4. Duleva, V. New aspects of food policy at European and national level, Social Medicine, 1, p.2-5, 2016.

5. WHO, Nutrition Policy, $<$ http://www.euro.who.int/en/healthtopics/diseaseprevention/nutrition/policy>

6. Gradinarova, N., Alexandrova, M, Public Health - Priority and Goal of the European Union, Collection of Summaries and Reports of the First National Conference "Public Health - Global Priority in Science and Practice" 9-10 June 2017, pp. 201-205

7. Popov, T., S. Garov, A. Enchev, Health Education in Cancer Patients. Thirty-nine Science and Technology Session ING collections ed. «TEMT»; Sofia, June 30, 2017, pp. 28-32. ISSN 1313-9134

8. Radev E, A. Yanakieva, R. Yaneva, Kr. Visev, M. Viseva, Principles of Human Resource Management in the Health Organization, International Journal of History and Philosophy of Medicine, Asklepios, Tom VIII (XXVII), Sofia, № 2, ed. East-West, pp. 81-84, 2013.

9. Brownell KD, Frieden TR. Ounces of prevention: the public policy case for taxes on sugared beverages. New Engl $J$ Med.360:1805-8, 2009.

10. Hector D, King L, Hardy L, et al. Evidence update on obesity prevention across the life course. Physical Activity Nutrition Obesity Research Group. 2012.

11.Naseva E., D. Shtereva, T. Kundurjiev. M, Methodological approach in studying the relationship between public health and its economic determinants, Health Policy and Management, Vol. 13, No. 1, pp. 38-43, 2013.

12. Obesity: guidance on the prevention, identification, assessment and management of overweight and obesity in adults and children (PDF). // National Institute for Health and Clinical Excellence (NICE).

13. Hill JO, Peters JC. Environmental contributions to the obesity epidemic. Science. 280:1371-3. 1998, PubMedCrossRefGoogle Scholar

14.Johns DJ, Hartmann-Boyce J, Jebb SA, et al. Diet or exercise interventions vs combined behavioral weight management programs: a systematic review and meta-
GORANOVA-SPASOVA R., et al. analysis of direct comparisons. $J$ Acad Nutr Diet. 114:1557-68, 2014.

15.Nutrition, Overweight and Obesity - EU Strategy, $\quad 2007<\mathrm{https}$ ://eurlex.europa.eu/legalcontent/BG/TXT/?uri $=\mathrm{L}$ EGISSUM:c11542c>

16.Duleva, C., Priorities in Nutrition in Europe, Proceedings of the $\mathrm{X}$ Congress on Nutrition - May, 2015.

17.Ministry of health, National Program for Prevention of Chronic Non-Communicable Diseases 2014-2020, <http://www.mh.government.bg/media/filer _public/2015/04/17/programa-preventsiyahronichni-nezarazni-bolesti-20142020.pdf>

18.Neseva E., M. Stoicheva, D. Shtereva, T. Kundurjiev. Indicators for the assessment of public health. Social medicine, num. 1, vol. 21, pp. 14-16, 2013.

19.Kass NE. An ethics framework for public health. Am J Public Health. 91:1776-82, 2001.

20. Vartanian LR, Smyth JM. Primum non nocere: obesity stigma and public health. $J$ Bioeth Inq. 10:49-57, 2013.

21.Azavedo, SM, Vartanian, LR, Ethical Issues for Public Health Approaches to Obesity, Curr, Obes Rep; 4:324-329, 2015.

22.Puhl RM, Heuer CA. The stigma of obesity: a review and update. Obesity. 17:941-64. 20098; PubMedCrossRefGoogle Scholar

23. Drewnowski A, Darmon N. Food choices and diet costs: an economic analysis. $J$ Nutr.;135:900-4, 2005.

24.Peycheva, K., R. Goranova, N. Gardinarova, L. Chakarova, Human Rights in Law, Religion and Medicine, Journal of Health Policy and Management, volume 17, № 4, ISSN 1313-4981, pp 53-59, 2017.

25.Andreyeva T, Long MW, Brownell KD. The impact of food prices on consumption: a systematic review of research on the prices elasticity of demand for food. Am J Public Health. 100:216-22, 2010.

26.ten Have M, de Beaufort ID, Teixeira PJ, et al. Ethics and prevention of overweight and obesity: an inventory. $O$

27.bes Rev. 12:669-79, 2011. 